\title{
The Difference in Kinematic Parameters During Gait Between the Patients with Knee Osteoarthritis and Healthy Controls Using an Insole with a Single Inertial Measurement Unit: A Case-Control study
}

Haruki Yaguchi ( $\square$ haruki.yaguchi.c4@tohoku.ac.jp )

Tohoku University Graduate School of Medicine

Keita Honda

Tohoku University Graduate School of Medicine

Yusuke Sekiguchi

Tohoku University Graduate School of Medicine

Huang Chenhui

NEC (Japan)

Fukushi Kenichiro

NEC (Japan)

Wang Zhenwei

NEC (Japan)

Nakahara Kentaro

NEC (Japan)

Masayuki Kamimura

Tohoku University School of Medicine

Takashi Aki

Tohoku University School of Medicine

Ken Ogura

Ogura Orthopaedic Clinic

Shin-Ichi Izumi

Tohoku University

\section{Research Article}

Keywords: Knee osteoarthritis, inertial measurement unit, gait analysis

Posted Date: March 2nd, 2022

DOI: https://doi.org/10.21203/rs.3.rs-1381570/v1

License: (c) (i) This work is licensed under a Creative Commons Attribution 4.0 International License. Read Full License 


\section{Abstract}

\section{Background:}

Gait disturbance is a critical problem in patients with osteoarthritis of the knee (KOA). The inertial measurement unit (IMU) is small size and lightweight allows for the measurement of patients without physical constraints. The purpose of this study was to investigate whether an insole with a single IMU can detect differences in kinematic parameters during gait between patients with KOA and healthy controls.

\section{Methods:}

Twenty patients with KOA, for whom unilateral total knee arthroplasty was planned, and 13 age-matched control subjects were included in this study. Participants wore shoes with IMU-equipped insoles and were required to walk across an 8-m walkway at a self-selected speed and foot movement data were collected during gait. We compared the spatiotemporal and kinematic parameters on the right side in healthy controls and both sides in patients with KOA.

\section{Results:}

The self-selected walking speed and stride length in the KOA group were significantly lower than those of the controls. The foot extension angular velocity at the toe-off (TO) was significantly smaller on the affected side than on the contralateral side $(p<0.001)$ and in the healthy controls $(p<0.001)$. During the swing phase, foot forward acceleration was significantly smaller on the affected side of KOA compared with healthy controls $(p=0.005)$. Furthermore, foot upward acceleration at the initial contact (IC) in patients with KOA was significantly lower on the contralateral side compared with that of healthy controls $(p=0.0167)$ but not on the affected side $(p=0.344)$, despite a decrease in walking speed.

\section{Conclusions:}

The insole with a single IMU can detect differences in foot kinematics during gait between patients with KOA and healthy controls. Our findings indicate that patients with KOA exhibit a dysfunction of the push-off at TO and shock absorption at the IC on the affected side.

\section{Introduction}

Knee pain during gait and gait disturbance are important issues in patients with osteoarthritis of the knee (KOA). Patients with KOA exhibit a smaller range of ankle, knee, and hip joint movements and a larger knee flexion moment in the sagittal plane than healthy controls, known as stiff-knee gait(1, 2). In the frontal plane, patients with KOA exhibit a larger knee adduction angle and adduction moment than healthy controls, known as lateral-thrust gait $(3,4)$. Furthermore, differences in knee kinematics and ankle, knee, and hip kinetics during gait have been reported with regards to the severity levels of KOA (5). The severity of KOA progresses due to abnormal knee joint loading during gait, and this progression is irreversible. Therefore, the quantification of gait disturbance plays an important role in detecting the progression of KOA and preventing irreversible changes.

Gait disturbance was quantified using an optical motion capture system. This system is the most accurate way to evaluate the kinematic and kinetic characteristics of gait (6). However, this system's measurements can only be conducted in a laboratory with infrared cameras, and many reflective markers must be attached to the landmarks of the body. Therefore, periodic evaluation of gait using an optical motion capture system is difficult for clinicians. Recently, the validity of kinematic gait analysis using multiple inertial measurement units (IMUs) has been 
demonstrated $(7,8)$, and several practical products have been developed (9-11). Because the IMU is small and lightweight, the subject can be measured without physical constraints. Furthermore, motion capture using multiple IMUs can measure gait outside the laboratory because the IMU can measure kinematics during gait simply by wearing it.

Ismailidiss (2021) reported that gait analysis using seven IMUs on the pelvis, thighs, shanks, and feet could detect differences in gait characteristics between patients with KOA and healthy controls (10). To continuously monitor gait in real life for progressive diseases such as KOA, it is important to reduce the number of IMUs and the opportunity to attach and detach them. If gait disturbance can be quantified using only an insole with a single IMU attached to the shoe, gait measurement is possible only when the patients wear shoes. Therefore, the use of an insole with a single IMU attached to the shoe may significantly reduce the daily effort required to attach the IMU. Previous studies using multiple IMUs $(10)$ and cameras $(5,12)$ have reported that the gait characteristics of patients with KOA were also observed from the kinematic parameters of the ankle joint. Therefore, it may be possible to detect the gait characteristics of patients with KOA by measuring foot movements using an insole with a single IMU.

The purpose of this study was to investigate whether an insole with a single IMU can detect differences in gait between patients with KOA and healthy controls. It is hypothesized that the foot angle and angular velocity in the sagittal plane are significantly smaller than those in healthy controls.

\section{Methods}

\section{Participants}

Participants were recruited from KOA patients who underwent medical examination at Tohoku University Hospital. The severity of KOA was assessed using the Kellgren-Lawrence $(\mathrm{KL})$ grading scale by an orthopedic surgeon $(0=$ no osteophytes, 1 = doubtful narrowing of joint space and possible osteophyte lipping, 2 = definite osteophytes and possible joint space narrowing, 3 = moderate multiple osteophytes, actual joint space narrowing, some sclerosis and possible bone contour deformity, 4 = large osteophytes, marked joint space narrowing, severe sclerosis, and definite bone contour deformity (13)). Healthy controls were subjected to asymptomatic age-matched people recruited from the local community via advertisements. The inclusion criteria for patients with KOA were (1) diagnosed with KOA by an orthopedic surgeon using radiography and (2) planning for unilateral total knee arthroplasty. The exclusion criteria for both groups were as follows: a history of any lesion or surgery that affected the lower limb or lumbar spine, a history of neurological disease that affected their gait, and a history of rheumatoid arthritis. All subjects provided informed consent, and the study was approved by the institutional ethics committee.

Twenty patients with KOA and 13 age-matched control subjects were included in this study (Table 1). The height of patients with KOA was significantly lower than that of healthy controls. Furthermore, body weight and body mass index were significantly higher in patients with KOA than in healthy controls. The side with the more severe symptoms, for which total knee arthroplasty is planned, was identified as the affected side. The KL grading scale on the affected side was 4 in all cases, while on the contralateral side, three patients had $\mathrm{KL}$ grading scale 2, three patients had $\mathrm{KL}$ grading scale 3 , six patients had $\mathrm{KL}$ grading scale 4, and eight patients had after total knee arthroplasty (Table 2 ). The control group had no pain in the lower or lumbar spine. 
Table 1

Demographic data for participants

\begin{tabular}{|c|c|c|c|}
\hline & KOA & Control & p-value \\
\hline $\mathrm{n}$ & 20 & 13 & \\
\hline Sex (Man: Female) & $2: 18$ & $4: 8$ & \\
\hline Age & $75.2(7.1)$ & 72(3.7) & 0.12 \\
\hline Height (cm) & $150.0(6.4)$ & $157.8(4.8)$ & $<0.01$ \\
\hline Weight (Kg) & $59.2(7.7)$ & $52(6.9)$ & $<0.01$ \\
\hline BMI & $26.3(2.7)$ & $20.7(2.2)$ & $<0.01$ \\
\hline \multicolumn{4}{|c|}{ Average (Standard deviation) } \\
\hline \multicolumn{4}{|c|}{ KOA: Knee Osteoarthritis, BMI: Body mass index } \\
\hline
\end{tabular}

Table 2

Kellgren-Lawrence grading scale for patients with knee osteoarthritis

\begin{tabular}{|lll|}
\hline KL grading scale & $\begin{array}{l}\text { Affected side } \\
\text { (More severe) }\end{array}$ & Contralateral side \\
\hline 0 & 0 & 0 \\
\hline 1 & 0 & 0 \\
\hline 2 & 0 & 3 \\
\hline 3 & 0 & 3 \\
\hline 4 & 20 & 6 \\
\hline post total knee arthroplasty & - & 8 \\
\hline KL grading scale: Kellgren-Lawrence grading scale
\end{tabular}

\section{Apparatus}

Gait data were collected using an IMU embedded within an insole (Fig. 1). The sensor (product dimensions: length, 40 $\mathrm{mm}$; width, $28 \mathrm{~mm}$; height, $6 \mathrm{~mm}$; weight: $9.6 \mathrm{~g}$ including battery) contains three accelerometers and three gyroscopes measuring foot motion with six degrees of freedom. Of the three accelerometer axes, the X-axis was defined as the left-right direction (left: +, right: -), the Y-axis as the anterior-posterior direction (posterior: +, anterior: -), and the Z-axis as the superior-inferior direction (superior: + , inferior: -). Each axis was programmed to sample at a rate of $100 \mathrm{~Hz}$. The data measured by the insole with a single IMU were transferred to and stored at the smartphone interface.

\section{Data Acquisition And Analysis}

Participants wore shoes with IMU-equipped insoles and were required to walk an 8-m walkway at a self-selected speed without a cane for five to seven trials. Foot movement was collected during gait using an insole with a single IMU operating at $100 \mathrm{~Hz}$. The data for the analysis were the average of five steps, excluding the first and last two steps. 
According to the manufacturer's proprietary software, the spatiotemporal and time-series data of foot kinematics were calculated based on the raw data measured by the insole with a single IMU (Huang et al.(14)). A foot angle of represents that the foot posture is horizontal to the floor. The positive value of the foot angle represents a posture in which the toe is more upward than the heel, which is defined as foot flexion. The negative value of the foot angle represents a posture in which the toe is more downward than the heel, which is defined as the foot extension. The initial contact (IC) and toe-off (TO) during gait were detected based on the peak value of the foot flexion angular velocity according to a previous study (Fig. 2) $(15,16)$. We calculated the foot flexion/extension angle and angular velocity, and foot forward and upward accelerations at IC and TO, and during the swing phase to understand the foot kinematics in the sagittal plane throughout the gait cycle. In patients with KOA, both affected and contralateral sides were employed. On the other hand, healthy controls adopted the data of the right leg.

\section{Statistical analysis}

Since we were particularly interested in the clinical relevance of side-to-side differences and group differences, we calculated $95 \%$ confidence intervals for within and between participants' differences in spatiotemporal and kinematic parameters. In addition, paired and independent samples t-tests were performed to detect statistically significant differences within and between participants, respectively. The significance level was set at 0.05 , and Bonferroni correction was performed to account for multiple comparisons, resulting in a significance level of 0.0175 .

\section{Results}

The self-selected walking speed and stride length in patients with KOA was significantly lower than those in healthy controls (walking speed: KOA $0.68 \pm 0.18 \mathrm{~m} / \mathrm{sec}$, healthy controls $1.08 \pm 0.17 \mathrm{~m} / \mathrm{sec}, \mathrm{p}<0.001$; stride length: KOA 0.88 $\pm 0.21 \mathrm{~m}$, healthy control $1.23 \pm 0.11 \mathrm{~m}, \mathrm{p}<0.001)$.

The foot flexion angle (affected side, $\mathrm{p}<0.001$; contralateral side, $\mathrm{p}<0.001$ ) and extension angular velocity (affected side, $p<0.001$; contralateral side, $p<0.001$ ) at IC were significantly smaller in patients with KOA than in healthy controls. Although the foot upward acceleration at IC was significantly smaller on the contralateral side in patients with KOA than in healthy controls $(p=0.0167)$, there was no significant difference between the affected side and healthy controls $(p=0.344)$ (Table 3$)$. 
Table 3

Foot kinematics at Initial contact

\begin{tabular}{|c|c|c|c|c|c|c|c|c|c|c|c|c|}
\hline \multirow[b]{2}{*}{$\begin{array}{l}\text { Foot angle } \\
\text { (degree) }\end{array}$} & \multicolumn{3}{|c|}{ Affected side } & \multicolumn{3}{|c|}{ Contralateral side } & \multicolumn{3}{|l|}{ Control } & \multirow{2}{*}{$\begin{array}{l}\mathbf{p 1} \\
< \\
0.001\end{array}$} & \multirow{2}{*}{$\begin{array}{l}\text { p2 } \\
<.001\end{array}$} & \multirow{2}{*}{$\begin{array}{l}\text { p3 } \\
0.341\end{array}$} \\
\hline & -1.22 & \pm & 4.78 & -2.75 & \pm & 5.87 & -10.46 & \pm & 5.00 & & & \\
\hline $\begin{array}{l}\text { Foot angular } \\
\text { velocity } \\
\text { (degree/sec) }\end{array}$ & 193.70 & \pm & 72.55 & 201.88 & \pm & 57.32 & 293.06 & \pm & 58.70 & $\hat{0}_{0.001}$ & $<_{0.001}$ & 0.405 \\
\hline $\begin{array}{l}\text { Foot forward } \\
\text { accelerations } \\
\left(\mathrm{m} / \mathrm{s}^{2}\right)\end{array}$ & 0.60 & \pm & 0.45 & 0.62 & \pm & 0.46 & 0.55 & \pm & 0.43 & 0.791 & 0.678 & 0.728 \\
\hline $\begin{array}{l}\text { Foot upward } \\
\text { accelerations } \\
\left(\mathrm{m} / \mathrm{s}^{2}\right)\end{array}$ & 1.63 & \pm & 0.63 & 1.41 & \pm & 0.43 & 1.83 & \pm & 0.46 & 0.344 & 0.0167 & 0.184 \\
\hline \multicolumn{13}{|c|}{ Average \pm Standard deviation } \\
\hline \multicolumn{13}{|c|}{ p1: p-value, Affected side vs. Control } \\
\hline \multicolumn{13}{|c|}{ p2: p-value, Contralateral side vs. Control } \\
\hline p3: p-value, Aff & ected side & vs. $C$ & ontrala & I side & & & & & & & & \\
\hline
\end{tabular}

The foot extension angle (affected side, $p=0.0125$; contralateral side, $p=0.005$ ), extension angular velocity (affected side: $p<0.001$, contralateral side: $p<0.001$ ), and foot forward acceleration (affected side, $p<0.001$; contralateral side, $p<0.001$ ) at TO were significantly smaller in patients with KOA than in healthy controls (Table 4). In addition, the foot extension velocity at TO was significantly lower on the affected side than on the contralateral side in patients with KOA $(p<0.001)$ (Table 4). 
Table 4

Foot kinematics at toe-off

\begin{tabular}{|c|c|c|c|c|c|c|c|c|c|c|c|c|}
\hline \multirow[b]{2}{*}{$\begin{array}{l}\text { Foot angle } \\
\text { (degree) }\end{array}$} & \multicolumn{3}{|c|}{ Affected side } & \multicolumn{3}{|c|}{ Contralateral side } & \multicolumn{3}{|l|}{ Control } & \multirow{2}{*}{$\begin{array}{l}\mathbf{p 1} \\
0.013\end{array}$} & \multirow{2}{*}{$\begin{array}{l}\text { p2 } \\
0.005\end{array}$} & \multirow{2}{*}{$\begin{array}{l}\text { p3 } \\
0.463\end{array}$} \\
\hline & 44.69 & \pm & 13.62 & 46.31 & \pm & 9.81 & 55.95 & \pm & 5.25 & & & \\
\hline $\begin{array}{l}\text { Foot angular } \\
\text { velocity } \\
\text { (degree/sec) }\end{array}$ & 311.72 & \pm & 92.39 & 347.74 & \pm & 86.84 & 520.09 & \pm & 51.47 & $\begin{array}{l}< \\
0.001\end{array}$ & $\begin{array}{l}< \\
0.001\end{array}$ & $\begin{array}{l}< \\
0.001\end{array}$ \\
\hline $\begin{array}{l}\text { Foot forward } \\
\text { accelerations } \\
\left(\mathrm{m} / \mathrm{s}^{2}\right)\end{array}$ & -0.73 & \pm & 0.56 & -0.90 & \pm & 0.41 & -2.07 & \pm & 1.00 & $\begin{array}{l}< \\
0.001\end{array}$ & 0.001 & 0.215 \\
\hline $\begin{array}{l}\text { Foot upward } \\
\text { accelerations } \\
\left(\mathrm{m} / \mathrm{s}^{2}\right)\end{array}$ & 1.26 & \pm & 0.23 & 1.37 & \pm & 0.28 & 1.18 & \pm & 0.33 & 0.476 & 0.115 & 0.152 \\
\hline \multicolumn{13}{|c|}{ Average \pm Standard deviation } \\
\hline \multicolumn{13}{|c|}{ p1: p-value, Affected side vs. Control } \\
\hline \multicolumn{13}{|c|}{ p2: p-value, Contralateral side vs. Control } \\
\hline p3: $p$-value, $A$ & sid & & tralate & ide & & & & & & & & \\
\hline
\end{tabular}

In the swing phase, foot flexion angular velocity was significantly lower in patients with KOA than in healthy controls (affected side: $p<0.001$, contralateral side: $p<0.001$ ). The foot forward acceleration in the swing phase was significantly smaller only on the affected side in patients with KOA than in healthy controls $(p=0.005)$. The foot upward acceleration was significantly larger in patients with KOA than in healthy controls (affected side, $p<0.001$; contralateral side, $p<0.001$ ) (Table 5). 
Table 5

Foot kinematics during swing phase

\begin{tabular}{|c|c|c|c|c|c|c|c|c|c|c|c|c|}
\hline \multirow[b]{2}{*}{$\begin{array}{l}\text { Foot angle } \\
\text { (degree) }\end{array}$} & \multicolumn{3}{|c|}{ Affected side } & \multicolumn{3}{|c|}{ Contralateral side } & \multicolumn{3}{|l|}{ Control } & \multirow{2}{*}{$\begin{array}{l}\text { p1 } \\
0.883\end{array}$} & \multirow{2}{*}{$\begin{array}{l}\text { P2 } \\
0.943\end{array}$} & \multirow{2}{*}{$\begin{array}{l}\text { p3 } \\
0.790\end{array}$} \\
\hline & 19.02 & \pm & 5.56 & 19.41 & \pm & 4.13 & 19.30 & \pm & 3.98 & & & \\
\hline $\begin{array}{l}\text { Foot angular } \\
\text { velocity } \\
\text { (degree/sec) }\end{array}$ & -97.92 & \pm & 25.17 & -97.92 & \pm & 25.17 & -136.01 & \pm & 16.37 & $\begin{array}{l}< \\
0.001\end{array}$ & $<_{0.001}$ & 0.085 \\
\hline $\begin{array}{l}\text { Foot forward } \\
\text { accelerations } \\
\left(\mathrm{m} / \mathrm{s}^{2}\right)\end{array}$ & 0.12 & \pm & 0.05 & 0.14 & \pm & 0.04 & 0.21 & \pm & 0.09 & 0.005 & 0.020 & 0.119 \\
\hline $\begin{array}{l}\text { Foot upward } \\
\text { accelerations } \\
\left(\mathrm{m} / \mathrm{s}^{2}\right)\end{array}$ & 0.81 & \pm & 0.08 & 0.78 & \pm & 0.07 & 0.63 & \pm & 0.06 & $\begin{array}{l}< \\
0.001\end{array}$ & $\iota_{0.001}$ & 0.038 \\
\hline \multicolumn{13}{|c|}{ Average \pm Standard deviation } \\
\hline \multicolumn{13}{|c|}{ p1: $p$-value, Affected side vs. Control } \\
\hline \multicolumn{13}{|c|}{ p2: p-value, Contralateral side vs. Control } \\
\hline \multicolumn{13}{|c|}{ p3: p-value, Affected side vs. Contralateral side } \\
\hline
\end{tabular}

\section{Discussion}

This study examined the differences in foot kinematics during gait between patients with KOA and healthy controls using an insole with a single IMU. The greater part of the foot kinematics at IC, TO, and during swing on the affected and contralateral sides in patients with KOA were significantly different from those in the healthy controls.

Considering the slower self-selected walking speed in patients with KOA, these changes may be influenced by KOA and decrease in walking speed. However, the foot extension angular velocity at TO on the affected side in patients with KOA was the smallest among the three sides. Furthermore, the foot forward acceleration during the swing phase was significantly smaller on the affected side compared with healthy controls. In addition, although the foot upward acceleration at IC was significantly lower on the contralateral side of patients with KOA than in the healthy controls, there was no significant difference between the affected side and the healthy controls, despite a decrease in walking speed. The foot extension angular velocity at TO, the foot forward acceleration during the swing phase, and the foot upward acceleration at IC may reflect the gait characteristics on the affected side in knee OA patients. These results support our hypothesis that foot movement during gait in patients with KOA differs from that in healthy controls. This is the first study to show that motion analysis using only the insole with a single IMU can detect the differences in gait kinematics between patients with KOA and healthy controls.

Our findings showed that the foot extension angular velocity at TO was smaller on the affected side in patients with KOA than in those on the contralateral side and in healthy controls. Furthermore, the foot forward acceleration in the swing phase was smaller only on the affected side in patients with KOA than in healthy controls. In normal walking in healthy subjects, the positive mechanical power generated by the gastrocnemius and soleus is generated during the late stance phase (including TO) and contributes to propel the lower limb during the swing phase (17). As a result, rapid plantar flexion at the ankle joint occurs in the late stance phase, called the push-off function. The slower foot extension angular velocity at TO and forward acceleration in the swing phase on the affected side in patients with KOA may reflect the dysfunction of the push-off. A previous study using a motion analysis system composed of seven 
IMUs reported that patients with KOA exhibited a smaller ankle plantar flexion angle at TO (10). Furthermore, a previous study using an optical motion analysis system with force plates reported that patients with KOA show a smaller forward acceleration of the whole-body center of mass (CoM) from late stance to pre-swing and slower gait speed compared to healthy controls (18). Our findings on foot kinematics at TO and the swing phase in patients with KOA are similar to that of previous studies. Therefore, motion analysis using the insole with a single IMU may be able to detect the characteristics of foot kinematics at TO and the swing phase for patients with KOA, as well as the motion analysis system with several IMUs.

The foot upward acceleration on the affected side at IC was not significantly different from that of healthy controls, although the self-selected walking speed was significantly slower in patients with KOA. In contrast, the foot upward acceleration on the contralateral side was significantly smaller than that in the healthy controls. In normal gait, the downward movement of the whole-body CoM is braked at the IC, resulting in an upward acceleration. A faster gait speed causes a greater impact force at the IC and an upward acceleration of the whole-body CoM $(19,20)$. The knee extensors play a role in absorbing the impact force after IC, which is called double knee action (21). Several previous studies have reported that double knee action is reduced in patients with KOA, resulting in a stiff-knee gait $(1,2)$. The non-significant decrease in the foot upward acceleration on the affected side, despite the decrease in walking speed, may reflect the dysfunction of shock absorption at the IC due to insufficient double knee action. A previous study using a portable force plate demonstrated a more significant ground reaction force in the early stance phase on the severe side of patients with KOA than the control with gait speed adjustment (22). Our findings of the foot upward acceleration at the IC on the contralateral side in patients with KOA are consistent with the previous result. Therefore, motion analysis using the insole with a single IMU may be able to detect the characteristics of IC in patients with KOA, as well as the optical motion capture system and multiple IMUs.

This study has several limitations. First, the differences in gait parameters between patients with KOA and healthy controls were not only due to the disease but also walking speed. Our findings showed side-to-side differences in the foot extension angular velocity at TO, foot forward acceleration in the swing phase, and foot upward acceleration at IC in patients with KOA. Therefore, these three parameters represent the characteristics of the affected side in patients with KOA. Second, the insole with a single IMU is currently unable to detect knee joint kinematics during gait in patients with KOA. However, in this study, the insole with a single IMU detected a smaller foot flexion angle at $\mathrm{HC}$ and a smaller foot extension angle at TO in patients with KOA than in healthy controls. Ro et al. (2019) showed that the range of ankle joint dorsiflexion/plantarflexion movement during the gait cycle was positively correlated with the range of knee flexion/extension movement in patients with KOA (12). Therefore, in the future, an insole with a single IMU might be able to estimate knee joint movement during gait in patients with KOA. Third, this study included patients with severe KOA. It is unclear whether the insole with a single IMU can detect the differences in gait parameters between patients with mild or moderate KOA and healthy controls. Furthermore, this study's results might be influenced by the functional status of the contralateral KOA, as the previous study reported that gait was different between unilateral and bilateral knee OA(23).

\section{Conclusions}

The insole with a single IMU can detect differences in foot kinematics during gait between patients with KOA and healthy controls. The foot extension angular velocity at TO was significantly smaller on the affected side than on the contralateral side and in healthy controls. Foot forward acceleration during the swing phase was significantly smaller only on the affected side than on the healthy controls. Furthermore, the foot upward acceleration at IC only on the contralateral side in patients with KOA was significantly lower than that in healthy controls, but not on the affected side, despite a decrease in walking speed. These findings represent a dysfunction of the push-off at TO and shock 
absorption at the IC on the affected side in patients with KOA. An insole with a single IMU has the potential to monitor longitudinal changes in gait more easily and naturally in daily life.

\section{List Of Abbreviations}

KOA

osteoarthritis of the knee

IMU

inertial measurement unit

TO

toe-off

IC

initial contact

$\mathrm{KL}$

Kellgren-Lawrence

CoM

center of mass

\section{Declarations}

\section{Ethics approval and consent to participate}

All subjects provided informed consent, and this study was approved by the Institutional Review Board of Tohoku University (approval ID: 2021-1-942) and was conducted according to the principles of the Declaration of Helsinki.

\section{Consent for publication}

Not applicable

\section{Availability of data and materials}

The datasets generated and/or analyzed during the current study are not publicly available but are available from the corresponding author on reasonable request.

\section{Competing interests}

C. Huang, K. Fukushi, Z. Wang, and K. Nakahara are employees of NEC corporation.

\section{Funding}

NEC Corporation supported this study.

\section{Authors' contributions}

Haruki Yaguchi: conception and design of the study, acquisition of data,

analysis and interpretation of data, drafting the article. Keita Honda and Sekiguchi Yusuke: conception and design of the study, acquisition of data, analysis and interpretation of data, revision of the article. Huang Chenhui, Fukushi Kenichiro, Wang Zhenwei, and Nakahara: analysis and interpretation of data, modification of the article. Masayuki 
Kamimura, Takashi Aki, and Ken Ogura: recruitment of patients, acquisition of data, revision of the article. Shin-Ichi Izumi: conception and design of the study, modification of the article. All authors approved the final manuscript.

\section{Acknowledgements}

We are grateful to Yuki Hayasaka for her help with data collection.

\section{References}

1. J. A. Gustafson, S. Gorman, G. K. Fitzgerald, S. Farrokhi, Alterations in walking knee joint stiffness in individuals with knee osteoarthritis and self-reported knee instability. Gait Posture 43, 210-215 (2016).

2. A. T. Collins, R. T. Richardson, J. S. Higginson, Interlimb symmetry of dynamic knee joint stiffness and cocontraction is maintained in early stage knee osteoarthritis. J Electromyogr Kinesiol 24, 497-501 (2014).

3. T. A. Gerbrands, M. F. Pisters, P. J. R. Theeven, S. Verschueren, B. Vanwanseele, Lateral trunk lean and medializing the knee as gait strategies for knee osteoarthritis. Gait Posture 51, 247-253 (2017).

4. P. C. Dixon, S. Gomes, R. A. Preuss, S. M. Robbins, Muscular co-contraction is related to varus thrust in patients with knee osteoarthritis. Clin Biomech (Bristol, Avon) 60, 164-169 (2018).

5. J. L. Astephen, K. J. Deluzio, G. E. Caldwell, M. J. Dunbar, C. L. Hubley-Kozey, Gait and neuromuscular pattern changes are associated with differences in knee osteoarthritis severity levels. J Biomech 41, 868-876 (2008).

6. P. J. Klein, J. J. DeHaven, Accuracy of three-dimensional linear and angular estimates obtained with the ariel performance analysis system. Archives of Physical Medicine and Rehabilitation 76, 183-189 (1995).

7. S. Park, S. Yoon, Validity Evaluation of an Inertial Measurement Unit (IMU) in Gait Analysis Using Statistical Parametric Mapping (SPM). Sensors (Basel) 21, 3667 (2021).

8. B. H. Kim et al., Measurement of Ankle Joint Movements Using IMUs during Running. Sensors (Basel) 21, 4240 (2021).

9. C. Nuesch, E. Roos, G. Pagenstert, A. Mundermann, Measuring joint kinematics of treadmill walking and running: Comparison between an inertial sensor based system and a camera-based system. J Biomech 57, 32-38 (2017).

10. P. Ismailidis et al., Side to side kinematic gait differences within patients and spatiotemporal and kinematic gait differences between patients with severe knee osteoarthritis and controls measured with inertial sensors. Gait Posture 84, 24-30 (2021).

11. K. Moerenhout, S. Chopra, X. Crevoisier, Outcome of the modified Lapidus procedure for hallux valgus deformity during the first year following surgery: A prospective clinical and gait analysis study. Clin Biomech (Bristol, Avon) 61, 205-210 (2019).

12. D. H. Ro et al., Effects of Knee Osteoarthritis on Hip and Ankle Gait Mechanics. Adv Orthop 2019, 9757369 (2019).

13. J. H. Kellgren, J. S. Lawrence, Radiological assessment of osteo-arthrosis. Ann Rheum Dis 16, 494-502 (1957).

14. C. Huang et al., Method for Estimating Temporal Gait Parameters Concerning Bilateral Lower Limbs of Healthy Subjects Using a Single In-Shoe Motion Sensor through a Gait Event Detection Approach. Sensors (Basel) 22, 351 (2022).

15. B. R. Greene et al., An adaptive gyroscope-based algorithm for temporal gait analysis. Med Biol Eng Comput 48, 1251-1260 (2010).

16. C. Wang et al., Real-Time Estimation of Knee Adduction Moment for Gait Retraining in Patients With Knee Osteoarthritis. IEEE Trans Neural Syst Rehabil Eng 28, 888-894 (2020). 
17. R. R. Neptune, S. A. Kautz, F. E. Zajac, Contributions of the individual ankle plantar flexors to support, forward progression and swing initiation during walking. J Biomech 34, 1387-1398 (2001).

18. S. Ogaya et al., Potential of muscles to accelerate the body during late-stance forward progression in individuals with knee osteoarthritis. Hum Mov Sci 61, 109-116 (2018).

19. T. S. Keller et al., Relationship between vertical ground reaction force and speed during walking, slow jogging, and running. Clinical Biomechanics 11, 253-259 (1996).

20. X. Wang, Y. Ma, B. Y. Hou, W. K. Lam, Influence of Gait Speeds on Contact Forces of Lower Limbs. J Healthc Eng 2017, 6375976 (2017).

21. D. A. Winter, Energy Generation and Absorption at the Ankle and Knee during Fast, Natural, and Slow Cadences. Clinical Orthopaedics and Related Research 175, 147-154 (1983).

22. K. E. Costello et al., Ground reaction force patterns in knees with and without radiographic osteoarthritis and pain: descriptive analyses of a large cohort (the Multicenter Osteoarthritis Study). Osteoarthritis Cartilage 29, 11381146 (2021).

23. M. W. Creaby, K. L. Bennell, M. A. Hunt, Gait differs between unilateral and bilateral knee osteoarthritis. Arch Phys Med Rehabil 93, 822-827 (2012).

\section{Figures}




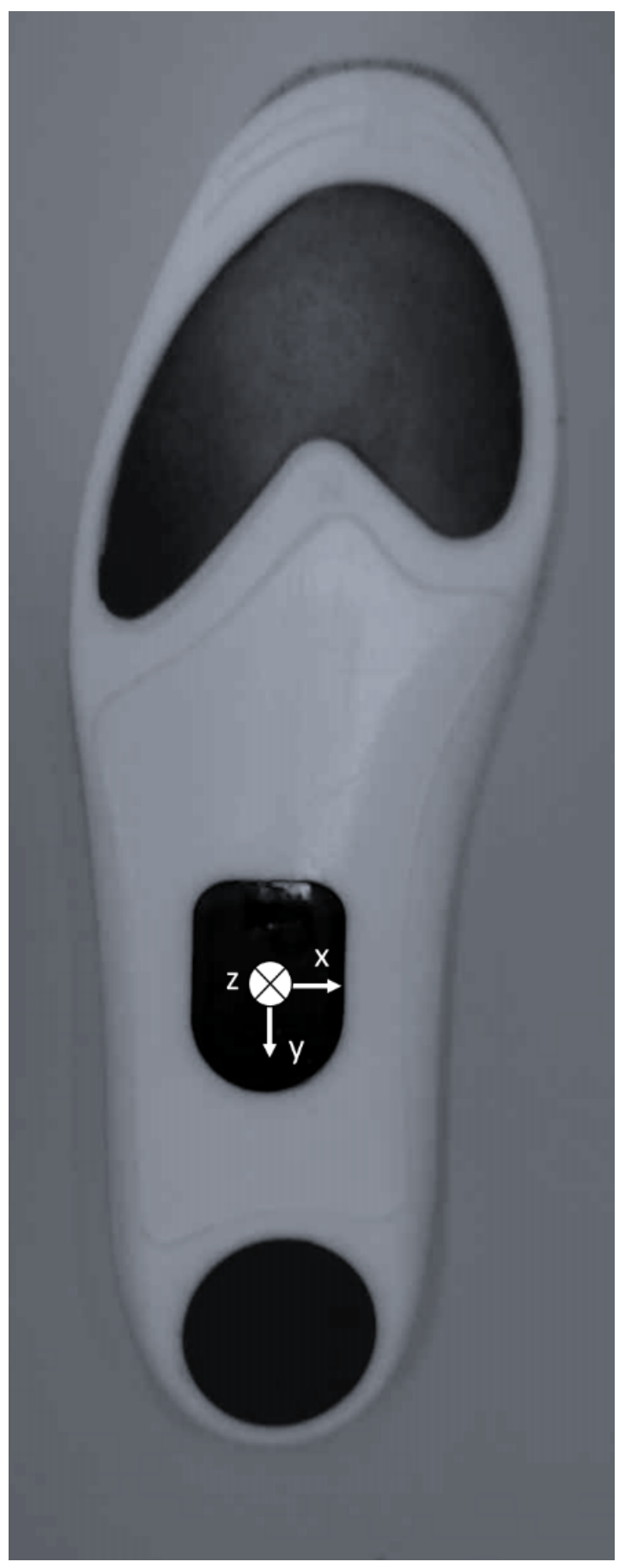

\section{Figure 1}

Inertial measurement unit (IMU) embedded within an insole

The IMU was mounted in the insole placed under the arch of the foot to prevent it from disturbing walking. 


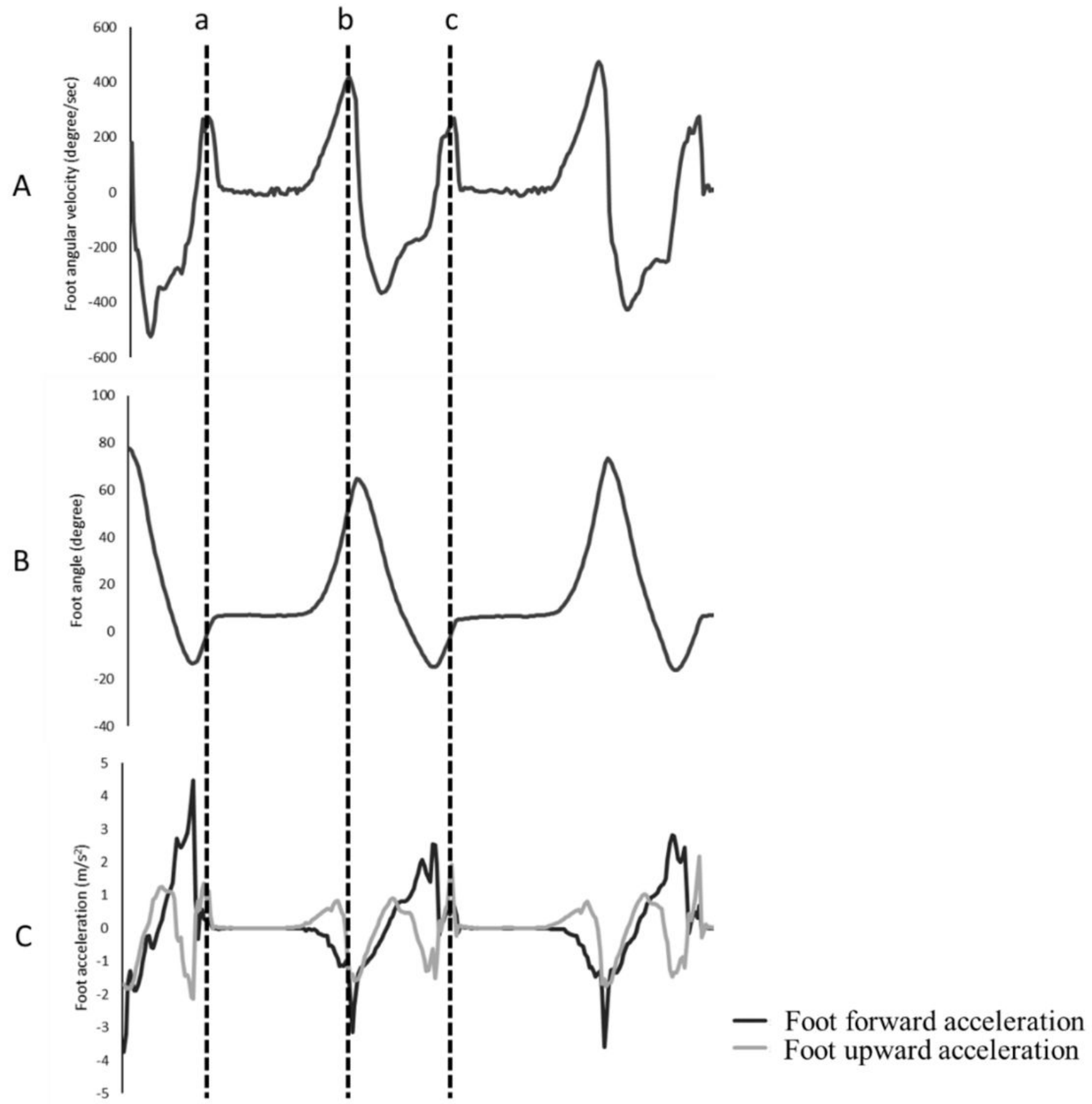

Figure 2

Inertial measurement unit signals of two consecutive gait cycles.

Data from the affected side in a patient of knee osteoarthritis.

A. Foot angular velocity, B. Foot angle, C. Foot acceleration

a and c. Initial contact, b. Toe-off

Stance phase: from a to b, Swing phase: from b to $c$, one gait cycle: from a to $c$ 